\title{
Arteriosclerosis in Dental Radiographs: Two Case Reports
}

\author{
Agnes Lau ${ }^{1,2}$ \\ ${ }^{1}$ Department of Oral and Maxillofacial Surgery, Massachusetts General Hospital, Boston, USA \\ ${ }^{2}$ Department of Oral and Maxillofacial Surgery, Harvard School of Dental Medicine, Boston, USA
}

\section{Email address:}

alau@partners.org

\section{To cite this article:}

Agnes Lau. Arteriosclerosis in Dental Radiographs: Two Case Reports. International Journal of Dental Medicine.

Vol. 5, No. 1, 2019, pp. 14-18. doi: 10.11648/j.ijdm.20190501.13

Received: January 18, 2019; Accepted: February 26, 2019; Published: March 19, 2019

\begin{abstract}
Dentists are trained to identify atheromatous plaques of the intimal layer of the common carotid artery on panoramic radiographs, but are less familiar with medial layer arteriosclerosis, which results in "pipe-stem" calcifications that may present on intraoral as well as extraoral dental radiographs. Medial arteriosclerosis is often found in diabetics and patients with chronic kidney disease, and is a strong predictor of stroke and cardiovascular events. Two case reports are presented that illustrate the presentation of medial arteriosclerosis in facial and maxillary arteries on dental radiographs. Dentists can play an important role in identifying patients at risk for stroke and cardiovascular events.
\end{abstract}

Keywords: Facial Artery, Vascular Calcification, Arteriosclerosis, Dental Radiograph

\section{Introduction}

Dentists can help with identifying vascular calcifications that are markers for cardiovascular events $[1,2]$. Dentists are trained in the identification of atheromatous plaques of the common carotid artery on panoramic radiography. Atherosclerosis is characterized by lipid accumulation, inflammation, fibrosis, plaque formation and calcification in the intimal layer of arteries that ultimately can obstruct the lumen of vessels or release emboli [3]. Common carotid artery calcifications detected on panoramic radiography have been shown to be powerful markers for future cardiovascular and cerebrovascular events $[1,2,4]$. They appear as circular or irregular radiopacities posterior to the angle of the mandible in the soft tissues of the neck, and their prevalence on panoramic radiography ranges from $6.9 \%$ in the general population to as high as $33.8 \%$ in patients with systemic diseases, such as Type 2 diabetes mellitus, hypertension, and hyperlipidemia [5-10].

There is another type of vascular calcification, medial artery calcification also known as Mönckeberg arteriosclerosis, that leads to a non-obstructive stiffening in the medial layer of arteries and decreased compliance of blood vessels [11]. The loss of arterial elasticity may lead to derangement in blood flow and injury to the endothelium, thus increasing the risk of thrombosis. As with intimal atherosclerosis, medial arteriosclerosis is associated with cardiovascular morbidity and mortality [11-13]. Medial artery calcification is related to age and structural changes from metabolic disease, such as diabetes and chronic kidney disease, and is independent of hypercholesterolemia [14, 15]. Medial artery sclerosis most often occurs in the extremities, but can also occur in the abdomen and head and neck areas $[16,17]$. Dentists can assist in the detection of this type of vascular calcification, as arteriosclerosis of the facial artery can present in routine dental radiographs [16, 18-20].

\section{Case Report 1}

A 75-year old white male presented to the Massachusetts General Hospital Dental Group for an initial outpatient comprehensive examination. His medical history was significant for the development of Type 1 diabetes mellitus at the age of 26 with resultant complications of peripheral vascular disease, peripheral neuropathy, autonomic neuropathy, retinopathy, toe amputation, and renal failure. He had poor chronic control and refused dialysis. He received a deceased donor left kidney and pancreas transplant at the age of 55. After the pancreatic transplant began to fail 13 years later, the patient was restarted on insulin. A repeat left kidney transplant was required at the age of 71.

The patient also had hypertension and hyperlipidemia, and 
a history of metabolic acidosis, deep vein thrombosis, and right leg angioplasty. He had no history of coronary artery disease, valvular disease, congestive heart failure, or arrhythmia. His medications included prednisone, tacrolimus, mycophenolate, insulin, furosemide, aspirin, and atorvastatin.

Clinical examination and a full mouth series of
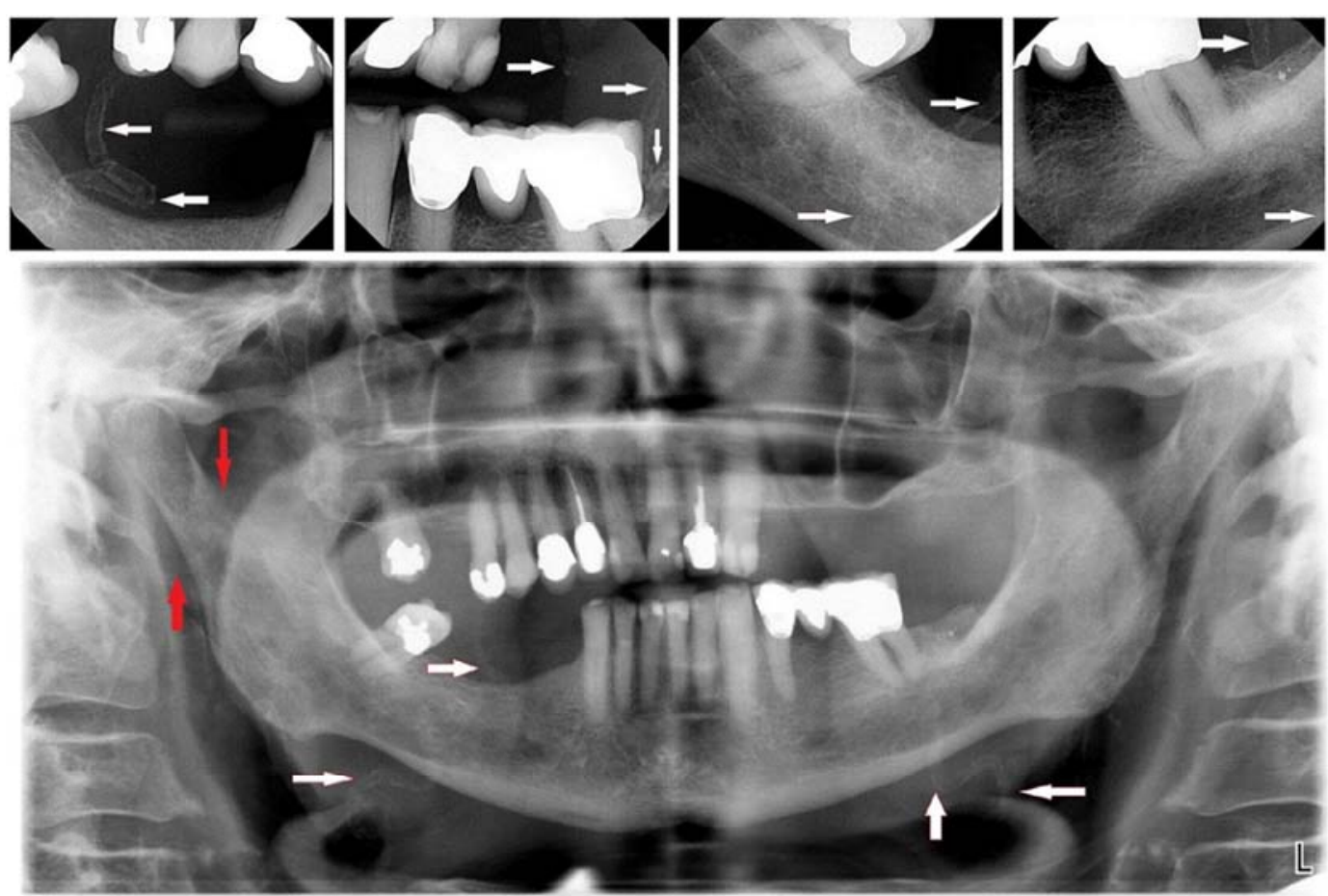

Figure 1. Case 1 - current bitewing, periapical, and panoramic radiographs revealing calcified bilateral facial (white arrows) and right maxillary (red arrows) arteries.

A panoramic radiograph was taken to better visualize the bilateral vascular calcification of the facial arteries (Figure 1). The panoramic radiograph also revealed a calcified right maxillary artery. Facial artery radiopacities were also evident radiographs revealed that the patient had partial edentulism and mild periodontitis. The radiographic exam was also significant for "pipe-stem" radiopacities following a tortuous track visible on bilateral bitewing and mandibular posterior periapical views (Figure 1).
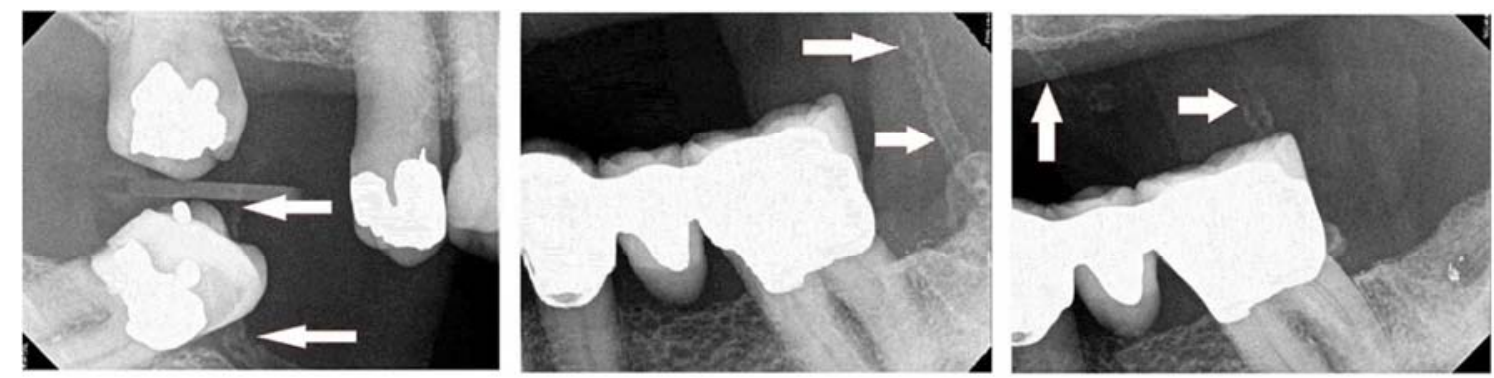

Figure 2. Case 1 - bitewing radiographs from 5 years previously revealing calcified bilateral facial arteries.
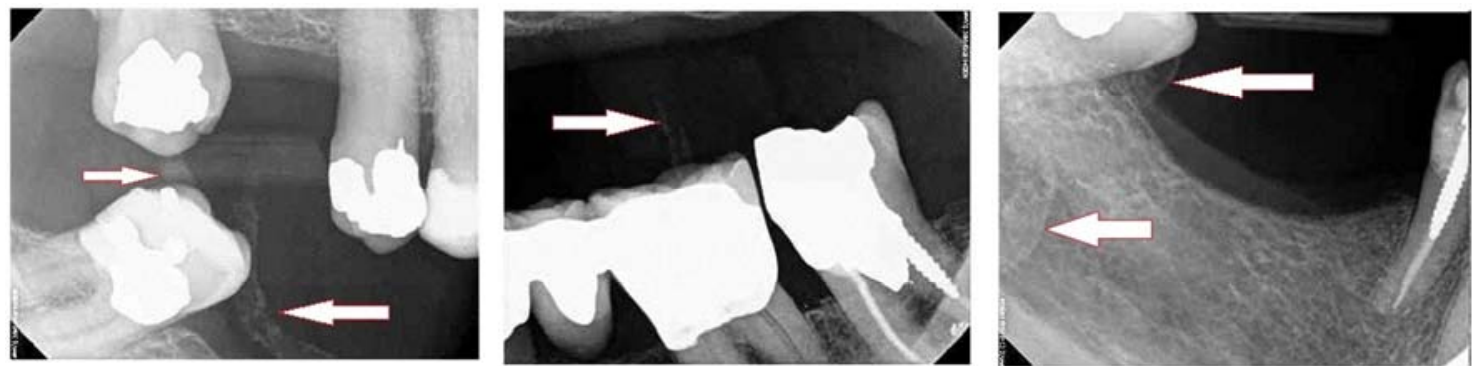

Figure 3. Case 1 - bitewing and periapical radiographs from 9 years previously revealing calcified bilateral facial arteries. 
The patient's primary care physician, nephrologist, and cardiologist were informed of the dental radiographic findings of progressive calcification of bilateral facial arteries. The patient was to return for routine dental treatment.

\section{Case Report 2}

A 29-year old male was admitted to the Massachusetts General Hospital for management of methicillin-sensitive Staphylococcus aureus bacteremia with a history of orthotopic liver transplant at 4 years of age for fulminant liver failure of unknown etiology, complicated by chronic rejection and tacrolimus-induced end-stage renal disease for the last 2 years. Other chronic conditions included hypertension and osteoarthritis. His medications included tacrolimus, prednisone, mycophenolate, sevelamer carbonate, amlodipine, lisinopril, valganciclovir, and vancomycin. $\mathrm{He}$ was on hemodialysis 3 days a week for the past year and was placed on the renal transplant waiting list. The current methicillin-sensitive Staphylococcus aureus infection was suspected to be from his hemodialysis line access, and a dental consultation was requested to rule out an oral source of infection.

On bedside examination, there was no extraoral asymmetry, lymphadenopathy, or limited range of motion. Intraoral examination revealed mild gingivitis and an MOD Ellis Class II fracture of the mandibular left second molar. A panoramic radiograph taken during admission revealed bilateral "pipe-stem" radiopacities following the tortuous tracks of the facial and maxillary arteries (Figure 4).

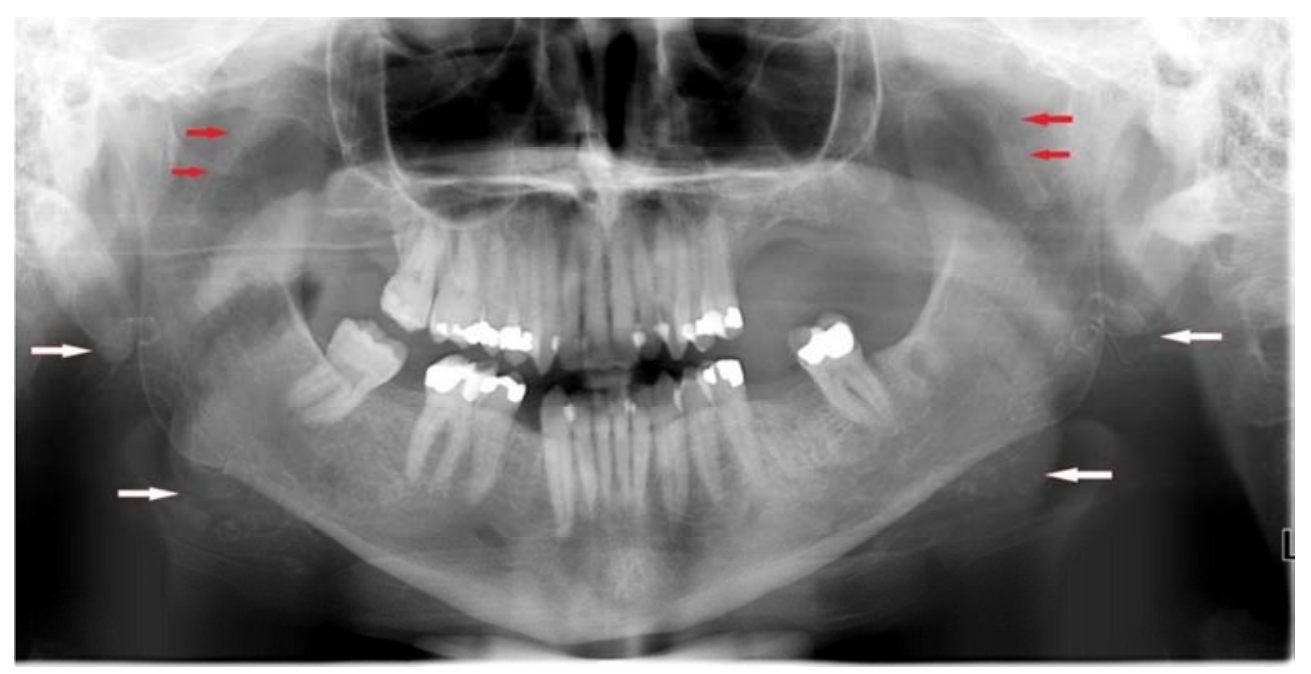

Figure 4. Case 2 - current panoramic radiograph revealing calcified bilateral facial (white arrows) and maxillary (red arrows) arteries.

The patient's medical team was informed of the lack of oral source of infection as well as the presence of calcification of the maxillary and facial arteries.

\section{Discussion}

These two cases illustrate medial artery calcification in the head and neck area in patients with chronic kidney disease. People with chronic kidney disease have multiple risk factors that induce vascular smooth muscle cells to transform into chondrocyte- or osteoblast-like cells, including abnormal bone metabolism that leads to high levels of circulating calcium and phosphorous, low levels of calcification inhibitors, impaired renal excretion, and side effects of therapies to treat renal osteodystrophy that accelerate arterial calcification $[11,21,22]$.

Medial artery calcification was first described as ring-like calcifications in a "pipe-stem" appearance by Lindblom in 1950 [23]. Medial artery calcifications detected by plain film have been reported in femoral, popliteal, anterior and posterior tibial, radial, and metacarpal arteries [19, 24]. Cases of facial artery medial calcification detected on intraoral radiographs were first published in 1942 by Ennis and 1966 by Hays $[16,18]$. Tahmasbi-Arashlow reported a case of $65-$ year old male with diabetes mellitus and end-stage renal disease on hemodialysis with panoramic and intraoral radiographic evidence of facial artery and lingual artery medial calcification [17]. From a review of 2,422 panoramic radiographs taken on Veterans Administration patients, 6 cases of facial artery calcification were found $(0.25 \%$ occurrence) by Miles with 4 of the 6 veterans having chronic renal failure, 2 of the 6 being on hemodialysis, and the age range of the 6 patients being 58-66 years [19]. Suarez reported a case of a 49-year old male with end-stage renal disease, cardiovascular disease, hyperparathyroidism, and renal osteodystrophy, whose panoramic radiograph revealed incidental findings of medial calcification of the facial, maxillary, and lingual arteries [20]. MacDonald reported a case of a 64-year old male with hypercalcemia and hyperphosphatemia from kidney disease, whose CT scan revealed medial calcification of not only the facial artery, but also the external carotid, lingual, internal maxillary, transverse facial, and superficial temporal arteries [25]. The patient profile of Case 1 in this report is typical in terms of 
age and medical issues, but the patient in Case 2 is unusual with respect to his young age, and to the best of this author's knowledge is the only published case of head and neck medial arteriosclerosis in a person in his twenties.

The common thread in most of these cases is chronic renal disease. Vipattawat determined that vascular calcification of any type can be detected on plain films in $57-81 \%$ of endstage renal disease patients and on CT scans in $60-70 \%$ of kidney transplant patients [22]. The prevalence of medial artery sclerosis in the general population was found to be $13.3 \%$ in males and $6.9 \%$ in females [24, 26]. Among populations at risk, medial artery calcification was found in $41.5 \%$ of Type 2 diabetics and $39.5 \%$ of end-stage renal disease patients [11]. With $9.4 \%$ of the U.S. population diagnosed with diabetes and $14 \%$ of the population diagnosed with chronic kidney disease, the prevalence of medial artery calcification is significant $[27,28]$.

Cardiovascular disease contributed to more than half of all deaths of patients with end-stage renal disease, and it has been shown that both intimal calcification and medial calcification of peripheral arteries are associated with increased mortality in these patients [13, 28]. Cardiovascular disease is also the leading cause of death in kidney transplant patients, accounting for $36-55 \%$ of deaths in patients with a functioning graft [29]. In kidney transplant patients the severity of vascular calcification increases with vintage of the transplant despite improvement in renal function and mineral metabolism [22]. Vascular calcification is a stronger predictor of cardiovascular events and all-cause mortality in kidney transplant patients than other risk factors [29].

Morbidity is also increased as affected arteries may not demonstrate a pulse. As a result, medial artery calcification is a significant risk factor for amputation in patients with chronic kidney disease and diabetes [30]. Diabetes and hypertension are the main causes of chronic kidney disease with almost half of chronic kidney disease patients having diabetes and/or cardiovascular disease [28]. Medial artery calcifications are not only observed in patients with advanced systemic disease. It has been shown that medial artery calcification in newly diagnosed non-insulin dependent diabetic patients is the most powerful predictive marker of future cardiovascular mortality even after adjustment for cardiovascular risk factors [31]. Therefore, recognition of medial artery calcification requires the institution of preventive measures and vigorous treatment where possible [11]. Dentists, and especially those working in medical settings where patients with significant health issues seek care, can play an important role in identifying patients at risk for cardiovascular events. Dentists are educated in identifying atheromatous plaques of the common carotid artery, but should also be aware of the presentation of medial arteriosclerosis in dental radiographs. They should know which populations are at high risk, but also understand that some patients who are much younger, as in Case 2, may present with advanced arteriosclerosis, and upon identification of such lesions make appropriate medical referrals $[4,20,32]$.

\section{Conclusion}

Vascular calcifications are associated with cardiovascular morbidity and mortality. It is important for dentists to be aware of dental radiographic evidence of medial arteriosclerosis in the patient population in general, and especially in patients at high risk, such as diabetics, patients with chronic renal disease, and renal transplant patients. Intraoral radiographs are taken every day in dental practice, allowing dentists to have a role in early detection of vascular calcification. Notification of medical providers and appropriate follow up may decrease the risks and complications of stroke and cardiovascular disease.

\section{Disclaimer}

The author declares no conflict of interest with regard to the research, writing, and publication of this study.

\section{Ethics Statement}

All radiographs and clinical information have been deidentified.

\section{References}

[1] M. Moshfeghi, J. B. Taheri, N. Bahemmat, M. E. Evazzadeh, and H. Hadian. Relationship between carotid artery calcification detected in dental panoramic images and hypertension and myocardial infarction. Iran J Radiol 2014 Aug; 11(3):e8714. doi: 10.5812/iranjradiol.8714. Epub 2014 Aug 1.

[2] A. H. Friedlander, S. M. El Saden, R. C. Hazboun, T. I. Chang, W. K. Wong, and N. R. Garrett. Detection of carotid artery calcification on the panoramic images of postmenopausal females is significantly associated with severe abdominal aortic calcification: a risk indicator of future adverse vascular events. Dentomaxillofac Radiol. 2015;447(7):20150094. doi: 10.1259/dmfr.20150094. Epub 2015 May 6.

[3] M. Rogers, C. Goettsch, and E. Aikawa. Medial and intimal calcification in chronic kidney disease: stressing the contributions. J Am Heart Assoc 2013;2:e000481. doi: 10.1161/JAHA.113.000481.

[4] J. Griniatsos, S. Damaskos, N. Tsekouras, C. Klonaris, and S. Georgopoulos. Correlation of calcified carotid plaques detected by panoramic radiograph with risk factors for stroke development. Oral Surg Oral Med Oral Pathol Oral Radiol Endod 2009; 108: 600-603.

[5] J. R. Goncalves, J. L. Yamada, C. Berrocal, F. H. Westphalen, A. Franco, and A. Fernandes. Prevalence of pathologic findings in panoramic radiographs: calcified carotid artery atheroma. Acta Stomatol Croat. 2016 Sept;50(3):230-234.

[6] U. K. Lee, T. I. Chang, N. Garrett, and A. H. Friedlander. Males with rheumatoid arthritis often evidence carotid atheromas on panoramic imaging: a risk indicator of future cardiovascular events. J Oral Maxillofac Surg. 2018 Jul;76(7):1447-1453. doi: 10.1016/j.joms.2018.01.006. Epub 2018 Jan 16. 
[7] Y. Atalay, F. Asutay, K. S. Agacayak, M. Koparal, F. Adali, and B. Gulsun. Evaluation of calcified atheroma on panoramic radiographs and Doppler ultrasonography in an older population. Clin Interv Aging. 2015 Jul 8;10:1121-1129. doi: 10.2147/CIA.S84683. eCollection 2015.

[8] N. Gustafsson, J. B. Ahlqvist, U. Näslund, P. Wester, K. Buhlin, A. Gustafsson, and E. Levring Jäghagen. Calcified carotid artery atheromas in panoramic radiographs are associated with a first myocardial infarction: a case-control study. Oral Surg Oral Med Oral Pathol Oral Radiol. 2018 Feb;125(2):199-204.e1. doi: 10:1016/j.oooo.2017.10.009. Epub 2017 Nov 15.

[9] N. Aghazadehsanai, T. I. Chang, N. R. Garrett, and A. H. Friedlander. Prevalence of calcified carotid artery atheromas on digital panoramic images among perimenopausal and postmenopausal African American women. Oral Surg Oral Med Oral Pathol Oral Radiol. 2017 May;123(5):621-625. doi: 10.1016/j.oooo.2017.01.011. Epub 2017 Feb 6.

[10] N. Alves, N. F. Deana, and I. Garay. Detection of common carotid artery calcifications on panoramic radiographs: prevalence and reliability. Int J Clin Exp Med 2014; 7(8): 1931-1939.

[11] P. Lanzer, M. Boehm, V. Sorribas, M. Thiriet, J. Janzen, T. Zeller, C. St. Hilaire, and C. Shanahan. Medial vascular calcification revisited: review and perspectives. Eur Heart J. 2014;35(23):1515-1525. doi: 10.1093/eurheartj/ehu163. Epub 2014 Apr 16.

[12] K. J. Rocha-Singh, T. Zeller, and M. R. Jaff. Peripheral arterial calcification: prevalence, mechanism, detection, and clinical implications. Catheter Cardiovasc Interv. 2014 May 1;83(6):E212-E220. doi: 10.1002/ccd.25387. Epub 2014 Feb 10.

[13] G. M. London, A. P. Guerin, S. J. Marchais, F. Metivier, B. Pannier, and H. Adda. Arterial media calcification in end-stage renal disease: impact on all-cause and cardiovascular mortality. Nephrol Dial Transplant 2003;18:1731-1740.

[14] N. Abou-Hassan, E. Tantisattamo, E. T. D’Orsi, and W. C. O'Neill. The clinical significance of medial arterial calcification in end-stage renal disease in women. Kidney Int. 2015 Jan;87(1):195-199. doi: 10.1038/ki.2014.187. Epub 2014 May 28.

[15] M. Rogers, C. Goettsch, and E. Aikawa. Medial and intimal calcification in chronic kidney disease: stressing the contributions. J Am Heart Assoc 2013;2:e000481. doi: 10.1161/JAHA.113.000481.

[16] J. B. Hays, J. A. Gibilisco, and J. L. Juergens. Calcification of vessels in cheek of patient with medial arteriosclerosis. Oral Surg 1966;24:299-302.

[17] M. Tahmasbi-Arashlow, S. Barghan, D. Kashtwari, and M. K. Nair. Radiographic manifestations of Mönckeberg arteriosclerosis in the head and neck region. Imaging Sci Dent 2016 ; 46;53-56.

[18] L. M. Ennis and L. W. Burket. Calcified vessels of the cheeks: demonstration by means of dental roentgenograms. Ann Dent 1942;1:111-113.

[19] D. A. Miles and R. M. Craig. The calcified facial artery: a report of the panoramic radiographic incidence and appearance. Oral Surg Oral Med Oral Pathol 1983;55:214219.

[20] M. M. Suarez-Cunqueiro, J. Duker, N. Leibehenschel, R. Schön, and R. Schmelzeisen. Calcification of the branches of the external carotid artery detected by panoramic radiography: a case report. Oral Surg Oral Med Oral Pathol Radiol Endod 2002;94:636-640.

[21] K. Nitta and T. Ogawa. Vascular calcification in end-stage renal disease patients. Contrib Nephrol. 2015;185:156-167. doi: 10.1159/000380980. Epub 2015 May 19.

[22] K. Vipattawat, C. Kitiyakara, B. Phakdeekitcharoen, S. Kantachuvesiri, V. Sumethkul, S. Jirasiritham, W. Stitchantrakul, and S. Disthabanchong. Vascular calcification in long-term kidney transplantation. Nephrology 2014;19:251256.

[23] A. Lindblom. Arteriosclerosis and arterial thrombosis in the lower limb. A roentgenological study. Acta Radiol. 1950;80(Suppl.):1-80.

[24] K. Kröger, A. Stang, J. Kondratieva, S. Moebus, E. Beck, A. Schmermund, S. Möhlenkamp, N. Dragano, J. Siegrist, K. Jöckel, and R. Erbel. Prevalence of peripheral arterial disease - results of the Heinz Nixdorf recall study. Eur J Epidemiol 2006;21:279-285.

[25] D. S. MacDonald, L. Zhang, and Y. Gu. Calcification of the external carotid arteries and their branches. Dentomaxillofacial Radiology 2012;41:615-618.

[26] R. G. Micheletti, G. A. Fishbein, J. S. Currer, and M. C. Fishbein. Mönckeberg sclerosis revisited: a clarification of the histologic definition of Mönckeberg sclerosis. Arch Pathol Lab Med 2008;132:43-47.

[27] Centers for Disease Control and Prevention. National Diabetes Statistics Report, 2017. Atlanta, GA: Centers for Disease Control and Prevention, U.S. Dept of Health and Human Services; 2017.

[28] Kidney Disease Statistics for the United States (December 2016). Retrieved from https://www.niddk.nih.gov/healthinformation/health-statistics/kidney-disease. Accessed March 24, 2018.

[29] G. Cianciolo, I. Capelli, M. L. Angelini, C. Valentini, O. Baraldi, M. P. Scolari, and S. Stefoni. Importance of vascular calcification in kidney transplant recipients. Am J Nephrol 2014;39:418-426.

[30] R. C. Johnson, J. A. Leopold, and J. Loscalzo. Vascular calcification: pathobiological mechanisms and clinical implications. Circ Res 2006;99;1044-1059.

[31] L. Niskanen, M. Suhonen, O. Siitonen, and M. Uusitupa. Medial artery calcification predicts cardiovascular mortality in NIDDM. Diabetes Care 1994;17:1-5.

[32] I. A. Monteiro, C. Ibrahim, R. Albuquerque, N. Donaldson, F. Salazar, and L. Monteiro. Assessment of carotid calcifications on digital panoramic radiographs: retrospective analysis and review of the literature. J Stomatol Oral Maxillofac Surg. 2018 Apr;119(2):102-106. doi: 10.1016/j.jormas.2017.11.009. Epub 2017 Nov 20. 